\title{
Analysis and Minimization of the Oscillatory Currents in Multibranch Thyristor-Switched Capacitors
}

\author{
Mohamed M. Saied \\ Electrical Engineering Department, College of Engineering and Petroleum, Kuwait University, P.O. Box 5969, Kuwait 13060, Kuwait \\ Correspondence should be addressed to Mohamed M. Saied, mmsaied2@yahoo.com \\ Received 18 May 2012; Revised 26 October 2012; Accepted 12 November 2012 \\ Academic Editor: Neville Watson \\ Copyright () 2012 Mohamed M. Saied. This is an open access article distributed under the Creative Commons Attribution License, \\ which permits unrestricted use, distribution, and reproduction in any medium, provided the original work is properly cited. \\ This paper addresses the switching transients in multibranch thyristor-switched capacitors (TSCs). The current transients \\ following the addition of a branch to a group of already connected ones are analyzed. Expressions for both its fundamental and its \\ oscillatory components are given in terms of the power network voltage, frequency, short-circuit level, and the switching angle. The \\ relations include also the compensator parameters such as its total reactive power rating, total number of branches, the number \\ of already connected branches, and the initial voltage on the capacitor involved in the switching transient. An expression for the \\ distortion of the supply current is also given. A minimization procedure is presented for identifying the optimal switching angle \\ leading to the least magnitude of the oscillatory current. Switching when the instantaneous supply voltage is equal to the initial \\ voltage will result in the least oscillatory current only in the two special cases of a single-branch compensator, or in the switching \\ of the first branch of a multi-branch TSC. The effect of both the total number of branches and the branch switching steps on the \\ oscillatory current and on the optimal switching angle is also discussed. The advantage of the suggested procedure is demonstrated \\ by investigating several case studies.
}

\section{Introduction}

There are four main practical applications of static Var compensators (SVCs) in electric power systems which can lead to considerable economical technical and/or economical advantages: to maintain the voltage at or near a constant level, to improve the power system stability, to improve the power factor, and, finally, to correct phase unbalances [1-11]. Most SVC's have branches comprising thyristor-switched capacitors (TSCs). A TSC includes several usually identical branches connected in parallel. By controlling the thyristors, the total capacitance and hence the capacitive reactive power delivered to the network can be changed. There are several factors affecting the choice of the number of branches in a TSC compensator $[2,4]$. In most practical applications, the number of branches, and hence of the control steps, lies between one and four [1]. Due to the switching-on and -off of these branches, the TSC terminal current includes unrequired high-frequency oscillatory components in addition to the useful capacitive current of the fundamental frequency. These components can lead to serious system disturbances.
Similar problems of capacitor switching transients related to the operation of distribution and transmission networks have been recognized in earlier investigations such as [610]. Reference [6] presented a comparative study of several suggested $600-\mathrm{V}$ or less 3 -phase switching configurations for minimal transients, while the study [7] was focusing on the transient overvoltages at the switched capacitors and other network locations in addition to the expected implications on the duties of the surge arresters and current limiting reactors.

The amplitudes and frequencies of the currents in a the multi-branch compensator depend mainly on the parameters of the power network supplying the TSC (primarily its operating voltage and short-circuit level), the total reactive power rating of the compensator, the total number of its branches, the switching time point of the TSC branch under consideration, and the number of already connected branches. A preliminary approach to the calculation of these currents and the identification of the optimal switching conditions for least oscillatory components was presented in the study [4]. The reference came to the main conclusion 


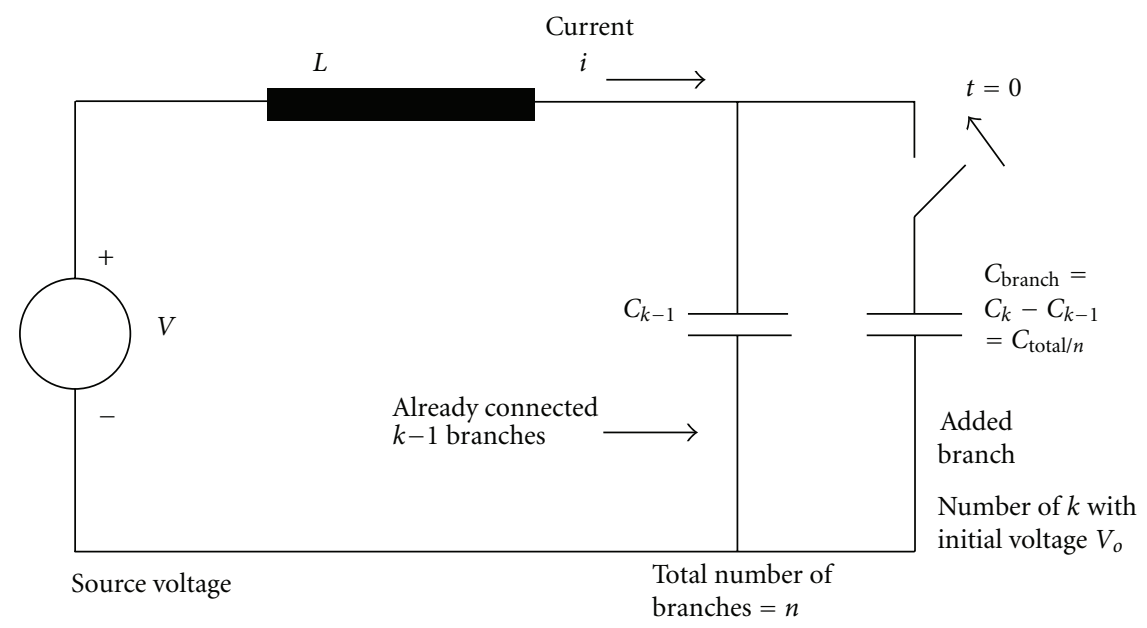

Figure 1: The equivalent circuit.

that the conditions for minimum oscillatory currents are close to the simple and nonideal condition of gating the thyristors when the instantaneous value of the supply voltage reaches the capacitor initial voltages. This statement implies the need for a more rigorous and generalized approach to the problem. Moreover, in order for the results to be more useful for practicing engineers, the current amplitudes, the time waveforms of the TSC total current and terminal voltage following each switching step, and the current distortion factor should be presented in a normalized way. They will be given in terms of the network's short-circuit level $S_{\mathrm{sc}}$ rather than its equivalent internal inductance $L$ and the TSC Var rating (preferably in per unit of $S_{\text {sc }}$ ) instead of its capacitance $C$. This will help recognize some general trends and implementation guidelines from the presented results.

\section{Method of Analysis}

Consider the equivalent circuit shown in Figure 1 representing one phase of a 3-phase TSC. It includes a voltage source given by $V_{m} \sin \left(\omega_{0} t+\alpha\right)$, where $V_{m}, \omega_{o}$, and $\alpha$ are its maximum phase value, angular frequency and its phase angle, respectively. It follows that at the switching time point $t=0$, the supply voltage assumes the instantaneous value $V_{m} \sin \alpha$. It can be shown that

$$
L=\frac{3 V_{m}^{2}}{\left(2 S_{\mathrm{sc}} \omega_{o}\right)} .
$$

It should be noted that the mentioned inductance is not a physically existing element. The equivalent inductance $L$ (having the power frequency reactance $\omega_{0} L$ ) in the circuit shown in Figure 1 represents the series connection of both the power network's short-circuit reactance (e.g., of a feeder or a cable section) as well as the leakage reactance of the eventually existing step-down power transformer.

$S_{\mathrm{sc}}$ is the network's 3-phase short-circuit power at the compensator terminals. The capacitor $C_{k-1}$ is the total capacitance of all already connected branches, while $\left(C_{k}-\right.$ $\left.C_{k-1}\right)=C_{\text {branch }}$ is the capacitance of the branch to be switched at $t=0$. If the total number of the identical branches is $n$, then each branch has a capacitance $C_{\text {branch }}=$ $C_{\text {total }} / n$. All these capacitance and inductance values are referred to the source side of the transformer, if any. It can be shown that

$$
C_{\text {total }}=\frac{2 Q_{\text {total }}}{\left(3 V_{m}^{2} \omega_{o}\right)},
$$

where $Q_{\text {total }}$ represents the total 3-phase reactive power delivered by the compensator if operated at $V_{m}$ and $\omega_{0}$. For convenience, the parameter $\gamma=Q_{\text {total }} / S_{\text {sc }}$ will be referred to as the per unit compensator reactive power rating. Each of the $n$ switching steps is given a number $k$ denoting the addition of the branch number $k$ in parallel with the $(k-1)$ already connected branches. From the sinusoidal steady state analysis, the following expressions for the initial values of the supply current, $I_{\text {initial }}$ and the voltage, $V_{\text {initial }}$ across the $(k-1)$ branches can be derived as follows:

$$
\begin{gathered}
I_{\text {initial }}=\frac{V_{m}}{\left[1 / \omega_{o} C_{k-1}-\omega_{o} L\right]} \sin \left[\alpha+\frac{\pi}{2}\right], \\
V_{\text {initial }}=\frac{\mathrm{V}_{m}\left(1 / \omega_{o} C_{k-1}\right)}{\left[1 / \omega_{o} C_{k-1}-\omega_{o} L\right]} \sin \alpha,
\end{gathered}
$$

where

$$
C_{k}=\frac{2 \gamma S_{\mathrm{sc}} k}{3 n V_{m}^{2} \omega_{o}} .
$$

Neglecting losses, the switching transient is described by the following differential equations subject to the above current initial condition:

$$
\begin{gathered}
V_{m} \sin \left(\omega_{o} t+\alpha\right)=L \frac{d i(t)}{d t}+v_{C_{k}}(t), \\
i(t)=C_{k} \frac{d v_{C_{k}}(t)}{d t} .
\end{gathered}
$$

The initial voltage across the capacitor $C_{k}$ can be determined from the initial voltage $V_{\text {initial }}$ across $C_{k-1}$ and the initial 
voltage $V_{o}$ across the switched new branch as $\left(V_{\text {initial }} C_{k-1}+\right.$ $\left.V_{o} C_{\text {total }} / n\right) / C_{k}$. It will be referred to as $V_{\text {eq }}$. The following solution for $i(t)$ and $v_{C_{k}}(t)$ could be obtained

$$
\begin{aligned}
& i(t)=\frac{1}{\sqrt{L}\left(-1+\omega_{o}^{2} L C_{k}\right)} \\
& \times\left[\sqrt{L}\left[I_{\text {initial }}\left(-1+\omega_{o}^{2} L C_{k}\right)+C_{k} \omega_{o} V_{m} \cos \alpha\right]\right. \\
& \times \cos \omega_{n, k} t-\sqrt{C_{k}} \\
& \times\left\{\sqrt{C_{k} L} \omega_{o} V_{m} \cos \alpha \cos \omega_{o} t\right. \\
& \quad+\left(-V_{\mathrm{eq}}+C_{k} L \omega_{o}^{2} V_{\mathrm{eq}}+V_{m} \sin \alpha\right) \\
&\left.\left.\quad \times \sin \omega_{n, k} t-\sqrt{C_{k} L} \omega_{o} V_{m} \sin \alpha \sin \omega_{o} t\right\}\right],
\end{aligned}
$$

$$
\begin{aligned}
v(t)= & \frac{1}{\sqrt{C_{k}}\left(-1+L C_{k} \omega_{o}^{2}\right)} \\
\times[ & -\sqrt{C_{k}} V_{m} \sin \alpha \cos \omega_{o} t \\
& +\sqrt{C_{k}}\left[\left(-1+L C_{k} \omega_{o}^{2}\right) V_{\mathrm{eq}}+V_{m}\right] \cos \omega_{n, k} t \\
& -I_{\text {initial }} \sqrt{L} \sin \omega_{n, k} t+C_{k} I_{o} L^{3 / 2} \omega_{o}^{2} \sin \omega_{n, k} t \\
& +C_{k} \sqrt{L} \omega_{o} V_{m} \cos \alpha \sin \omega_{n, k} t \\
& \left.-\sqrt{C_{k}} V_{m} \cos \alpha \sin \omega_{o} t\right] .
\end{aligned}
$$

Both the current and the capacitor voltage will contain higher frequency parts of the angular frequency $\omega_{n, k}=1 / \sqrt{L C_{k}}$ in addition to the power frequency components.

Focusing on the current, its power frequency component has the waveform $I_{\text {fund,max }} \operatorname{Cos}\left(\omega_{0} t+\alpha\right)$. Its amplitude is $I_{\text {fund,max }}=\omega_{o} V_{m} C_{k} /\left(1-\omega_{o}^{2} L C_{k}\right)$. It can be seen that the oscillatory component follows the equation:

$$
i_{\mathrm{osc}}(t)=A \sin \omega_{n, k} t+B \cos \omega_{n, k} t,
$$

where $A$ and $B$ can be easily determined from the expression of the current waveform in (6) as:

$$
\begin{aligned}
A=-\sqrt{\frac{C_{k}}{L}}( & -V_{o}+L \omega_{o}^{2} V_{o} C_{k}+V_{m} \sin \alpha \\
- & {\left[\left(C_{k-1}\left(-1+L \omega_{o}^{2} C_{k}\right)\left(-V_{o}+V_{o} L \omega_{o}^{2} C_{k-1}\right)\right.\right.} \\
& \left.\left.\left.+V_{m} C_{k} \sin \alpha\right) /\left(-1+L \omega_{o}^{2} C_{k-1}\right) C_{k}\right]\right)\left(-1+L \omega_{o}^{2} C_{k}\right)^{-1}, \\
B= & \frac{\omega_{o} V_{m}\left(C_{k-1}-C_{k}\right)}{\left(-1+L \omega_{o}^{2} C_{k-1}\right)\left(-1+L \omega_{o}^{2} C_{k}\right)} \cos \alpha,
\end{aligned}
$$

the amplitude of the oscillatory current is therefore $I_{\text {osc }}=$ $\sqrt{A^{2}+B^{2}}$.

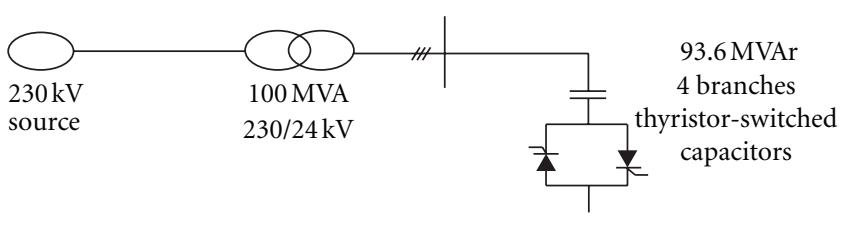

FIgURE 2: Sample network.

From (9), the main factors affecting the amplitude of the oscillatory current are the series inductance, the compensator reactive power rating, the total number of its branches $n$, the initial voltage $V_{o}$ of the switched branch of the number $k$, and the switching angle $\alpha$.

The results of applying the above expressions to the special case of a single branch $(n=1)$ agree with those available in the literature.

The optimal switching angle $\alpha$ leading to the least amplitude of the oscillating current $I_{\text {osc }}$ can be determined from

$$
\frac{d\left(A^{2}+B^{2}\right)}{d \alpha}=0 .
$$

\section{Sample Results}

The procedure is applied to the network shown in Figure 2, with the following data $[1,4]$.

Rated line voltage $=230 \mathrm{kV}$.

Rated frequency $=60-\mathrm{Hz}$.

Total compensator capacitive reactive power $=$ 93.6 Mvar.

Number of identical branches $=4$.

Assumed short-circuit level at the TSC terminals, taking into account the transformer reactance $S_{\mathrm{sc}}=$ 900 MVA.

The results depicted in Figure 3 are computed for an initial branch capacitor voltage equal to the amplitude of the rated network phase voltage $V_{m}$ and a switching angle of $\alpha=90^{\circ}$. Only integer values on the $x$-axes (switching step $k$ ) are meaningful. The plots show the conditions after switching the $k$ th branch. Plot (a) indicates that the amplitude of the fundamental current is almost proportional to the number $k$ denoting the switching step. The slight deviation from the linear relationship is due to the voltage drop across the series inductance, which depends on $k$. Plots (b) and (c) give the amplitudes and frequencies (as multiples of the supply frequency $60-\mathrm{Hz}$ ) of the oscillatory current component following each switching step. Beyond $k=2$, the oscillatory current increases less than proportional with $k$. Its frequency drops from 6.2 times the supply frequency upon connecting the first branch $(k=1)$ to half this value (3.1 per unit) for $k=4$, while adding the last (fourth) branch to the already connected three. This is because the capacitance included in the equivalent circuit in the first case is one fourth of that in the case. Plot (d) illustrates the current distortion defined as the oscillatory current amplitude in 


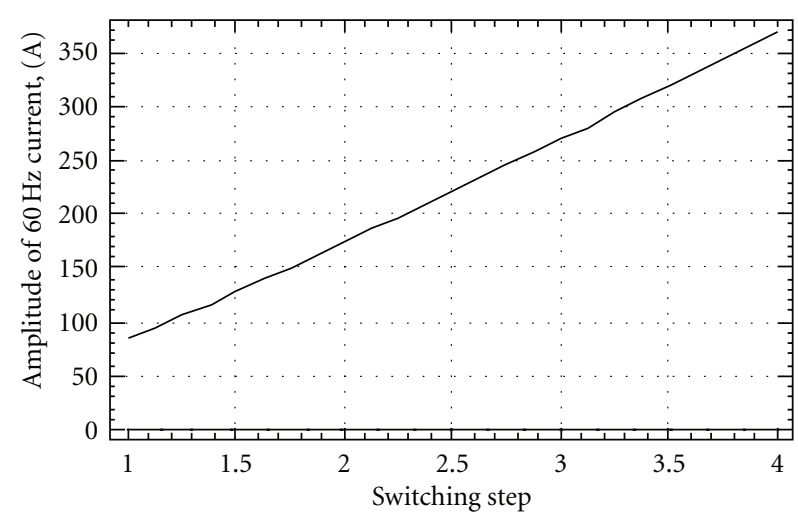

(a)

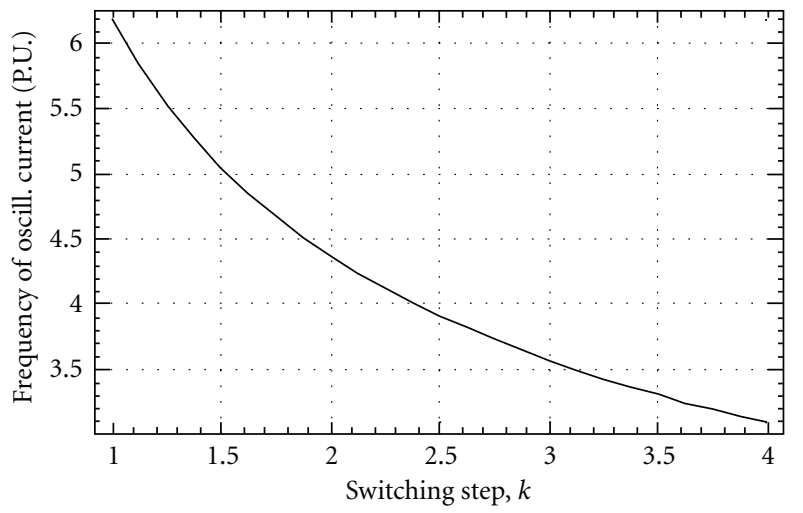

(c)

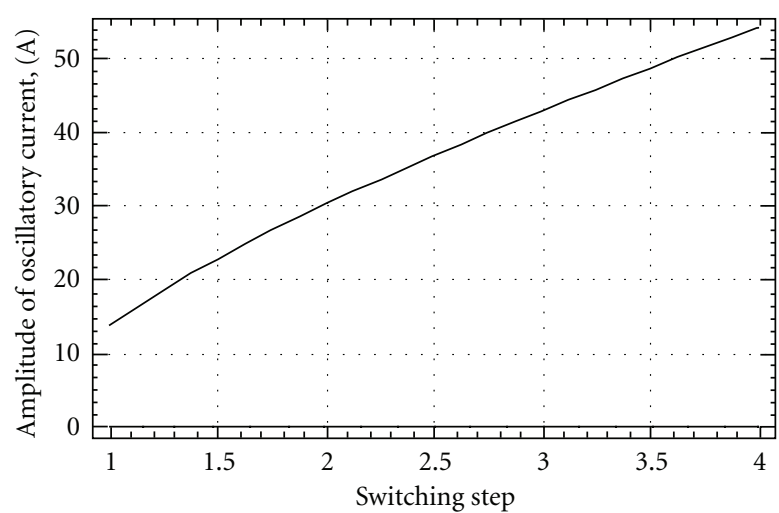

(b)

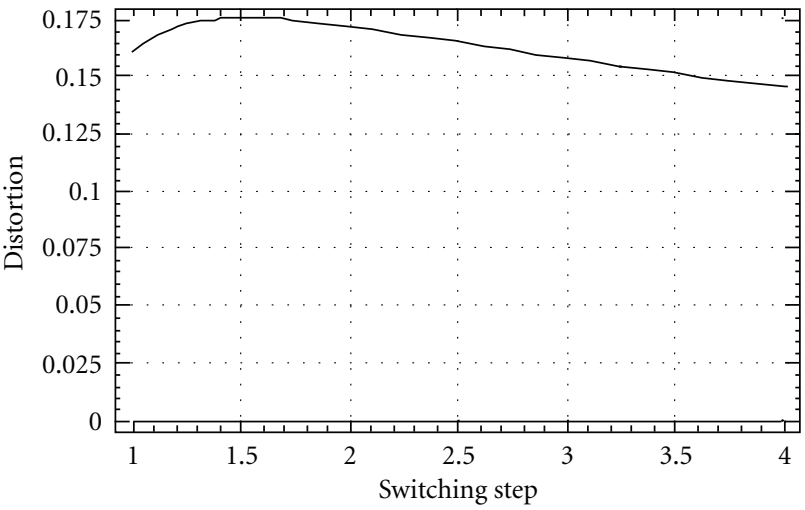

(d)

Figure 3: The fundamental and oscillatory currents for the different switching steps $k$. The initial branch capacitor voltages $V_{o}=V_{m}$ and the switching angle $\alpha=90^{\circ}$.

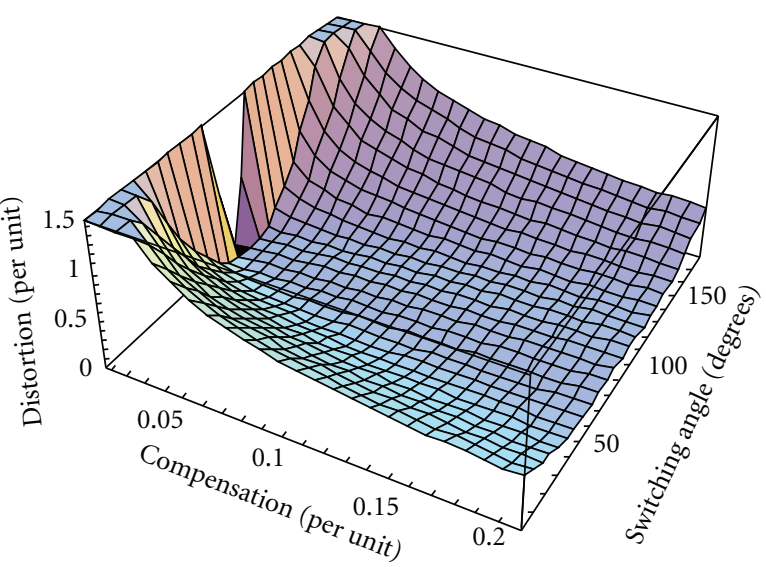

FIGURE 4: The current distortion as a function of both the per unit compensator rating and the switching angle $\alpha$.

per unit of the fundamental current, that is, $I_{\text {osc }} / I_{\text {fund,max }}$. Its largest value of about 0.170 occurs while switching the second branch, and the least value is 0.146 and corresponds to $k=4$.

The 3-dimensional plot in Figure 4 shows the dependence of the distortion factor of the last switching step $(k=4)$ on both the total per unit reactive power rating of the compensator $\gamma$ and the switching angle $\alpha$, assuming an initial voltage $V_{o}=V_{m}$ for the branch capacitors.
It indicates the general trend that the current distortion decreases with the compensator rating. It further shows that for small compensator ratings the least distortion occurs if the switching takes place at a switching angle close to $\alpha=$ $90^{\circ}$, while for larger ratings the minimum distortion can occur over a wider range of $\alpha$.

The impact of the initial voltage $V_{o}$ together with the switching angle $\alpha$ on the current distortion is depicted in Figure 5. It shows that, for small initial capacitor voltages, 


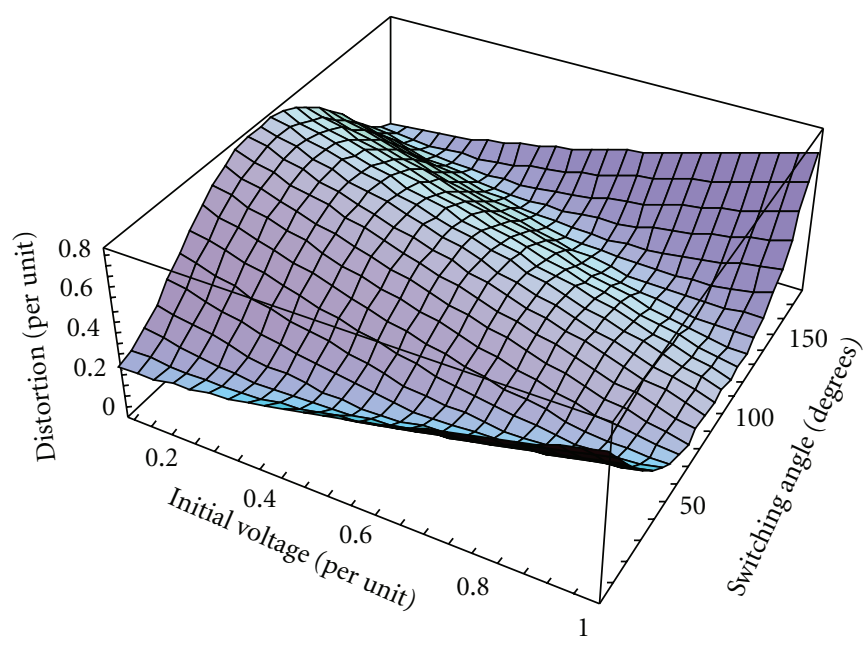

Figure 5: The current distortion versus the per unit value of the initial capacitor voltage $z=V_{o} / V_{m}$ and the switching angle $\alpha$.

the least distortion occurs at the switching angle $\alpha=0$, and the largest distortion results if $\alpha=90^{\circ}$. For initial capacitor voltages close to $V_{o}=V_{m}$, however, the largest distortion occurs at $\alpha=0$. The least distortion will result if $\alpha=67.2^{\circ}$.

The optimization procedure based on (10) will be demonstrated by considering the example of a 4-branch compensator having a per unit compensation level $\gamma=0.104$. By direct differentiation with respect to $\alpha$, the following expression can be obtained for the switching of the branch number $k$ to the already connected $(k-1)$ branches with an initial capacitor voltage of $V_{o}=z \cdot V_{m}$ as follows:

$$
\begin{aligned}
& \left(\frac{1}{I_{\text {fund,max }}^{2}}\right) \frac{d\left(A^{2}+B^{2}\right)}{d \alpha} \\
& =\left(4.257 \times 10^{-69}\left(1.8 \times 10^{9}-4.68 \times 10^{7} k\right)^{2}\right. \\
& \times \cos \alpha \cdot\left(-1.0597 \times 10^{39}(-39.4615+k)\right. \\
& \times(-38.4603+k)\left(7.786 \times 10^{15}+k\right) \\
& \times\left(1479.34-76.9243 k+k^{2}\right) z \\
& +2.0134 \times 10^{26}\left(-1.576 \times 10^{30}+k\right) \\
& \times(-38.4614+k)\left(1479.3-76.9232 k+k^{2}\right) \\
& \times \sin \alpha)) /\left((-39.4615+k)^{2}(-38.4615+k)^{4}\right. \\
& \left.\quad \times\left(1 \times 10^{-25}+k\right)\left(1 \times 10^{-6}+k\right)^{2}\right) .
\end{aligned}
$$

Equating this first derivative to zero will give the following three roots:

$$
\begin{gathered}
\alpha= \pm \frac{\pi}{2}, \\
\alpha=\sin ^{-1} 10^{13} \\
\cdot\left[z \cdot \left(3.37 \times 10^{22}-3.48 \times 10^{21} k+1.35 \times 10^{20} k^{2}\right.\right. \\
\left.-2.32 \times 10^{18} k^{3}+1.50 \times 10^{16} k^{4}+1.93 k^{5}\right) \\
\times\left(3.28 \times 10^{35}-2.56 \times 10^{34} k+6.66 \times 10^{32} k^{2}\right. \\
\left.\left.-5.77 \times 10^{30} k^{3}+3.66 k^{4}\right)^{-1}\right] .
\end{gathered}
$$

As will be seen later, the third root will result in the global minimum of the current distortion. It can be seen that, for all switching steps $k$, the value of $\sin \alpha$ is proportional to the per unit initial voltage of the switched capacitor $z$.

The three plots (a), (b), and (c) in Figure 6 give the optimal switching angle $\alpha$ for the total compensator ratings: $\gamma=0.03,0.104$ and 0.208 per unit, respectively. The four curves in each plot give the optimal angle $\alpha$ for the switching of the first branch $k=1$ (the upper curve), $k=2, k=3$, and $k=4$ (the lowest curve). The upper curves for switching the first branch $(k=1)$ in the three plots (a), (b), and (c) are identical, and they follow the known relation of a single-branch TSC: $\sin \alpha=V_{o} / V_{m}$. They all indicate that the optimal $\alpha$ is $90^{\circ}$ for $V_{o}=V_{m}$. For the switching of the three other branches, the optimal angle yielding the least distortion will be smaller than $\sin ^{-1}\left(V_{o} / V_{m}\right)$. The deviation increases with $k$ and with the capacitor initial voltage $V_{o}=z \cdot V_{m}$.

Table 1 gives the optimal switching angle $\alpha$ in degrees corresponding to the four switching steps $k$, with the capacitor initial voltages $V_{o}=V_{m}$, that is, $z=1$. They are calculated for three different values of the per unit compensator rating $\gamma: 0.030,0.104$, and 0.208 . The results show that the optimal switching angle decreases with the total per unit rating of the compensator $\gamma$ and with the number $k$ denoting the switching step. As known from single-branch TSC, the optimal value of $\alpha$ for the first step $(k=1)$ is $90^{\circ}$ for all per unit compensator ratings $\gamma$.

Table 2 shows the fundamental and oscillatory current components as well as the current distortion factor if the optimal values of the switching angle are used for the four switching steps for a compensator per unit rating $\gamma=0.104$. Both current components increase approximately proportional to the step number $k$. The distortion factor ranges between 0.163 per unit for $k=2$ and 0.132 for the last step $k=4$. These values are less than the corresponding ones $(0.173$ per unit for $k=2$ and 0.146 for the last step $k=4)$ in Table 3 presenting the results if the switching angle $\alpha=90^{\circ}$ is applied. By comparing Tables 2 and 3, it can be realized that using the optimal values of $\alpha$ will result in 
TABLE 1: The optimal switching angles $\alpha(z=1)$.

\begin{tabular}{lccc}
\hline Compensator step rating $\rightarrow$ & $\gamma=0.030$ per unit & $\gamma=0.104$ per unit & $\gamma=0.208$ per unit \\
\hline$k=1$ & 90 & 90 & 90 \\
$k=2$ & 83 & 77 & 71.44 \\
$k=3$ & 80 & 71.5 & 63.64 \\
$k=4$ & 78 & 67.2 & 57.57 \\
\hline
\end{tabular}

TABLE 2: The fundamental and oscillatory currents and the current distortion factor while applying optimal switching angles $\alpha(\gamma=0.104$, $z=1$ ).

\begin{tabular}{lccc}
\hline Switching step & Fundamental current, A & Oscillatory current, A & Current distortion, per unit \\
\hline$k=1$ & 85.287 & 13.752 & 0.161 \\
$k=2$ & 175.253 & 28.543 & 0.163 \\
$k=3$ & 270.292 & 39.346 & 0.146 \\
$k=4$ & 370.847 & 49.089 & 0.132 \\
\hline
\end{tabular}

TABLE 3: The fundamental and oscillatory currents and the current distortion factor while applying switching angle $\alpha=90^{\circ}(\gamma=0.104, z=$ $1)$.

\begin{tabular}{lccc}
\hline Switching step & Fundamental current, A & Oscillatory current, A & Current distortion, per unit \\
\hline$k=1$ & 85.287 & 13.752 & 0.161 \\
$k=2$ & 175.253 & 30.240 & 0.173 \\
$k=3$ & 270.292 & 42.858 & 0.159 \\
$k=4$ & 370.847 & 54.220 & 0.146 \\
\hline
\end{tabular}

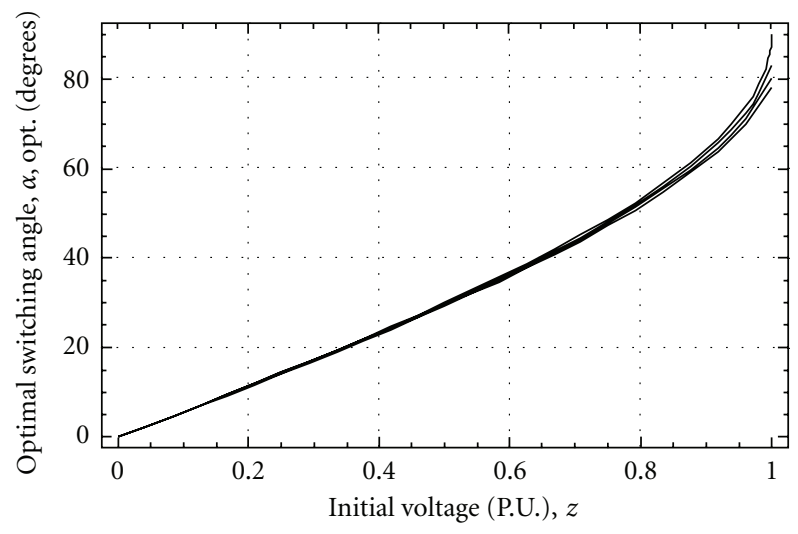

(a) Compensator rating $\gamma=0.03$ per unit

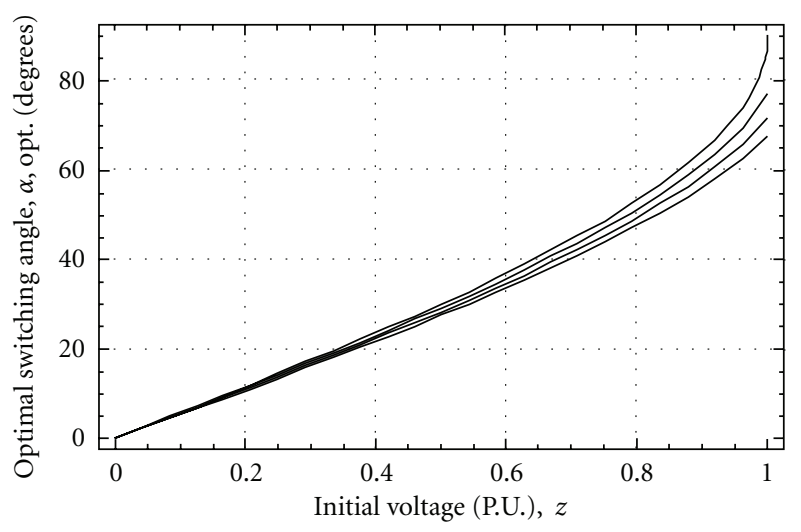

(b) Compensator rating $\gamma=0.104$ per unit

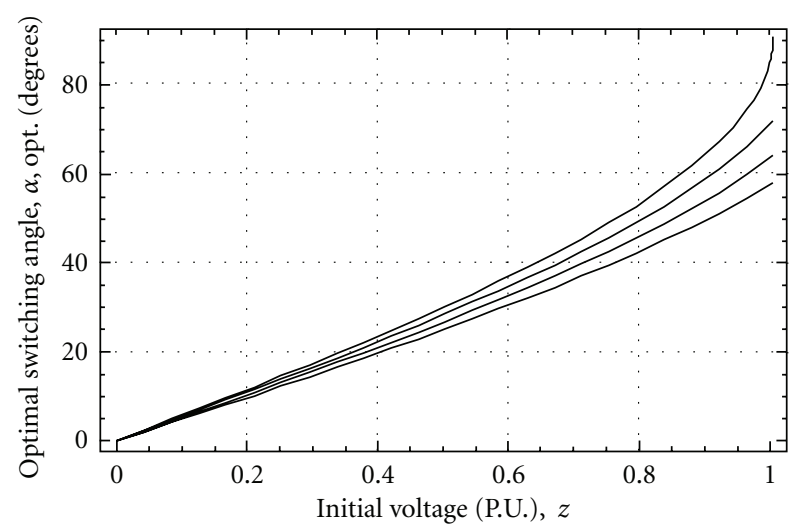

(c) Compensator rating $\gamma=0.208$ per unit

FIGURE 6: The optimal switching angle $\alpha$ as a function of the per unit capacitor initial voltage $z=V_{o} / V_{m}$, for switching each of the four branches $k=1,2,3$ and 4 . Plots for three different per unit total compensator ratings are shown. In each plot, the top curve corresponds to $k=1$, and the bottom curve applies for $k=4$. 


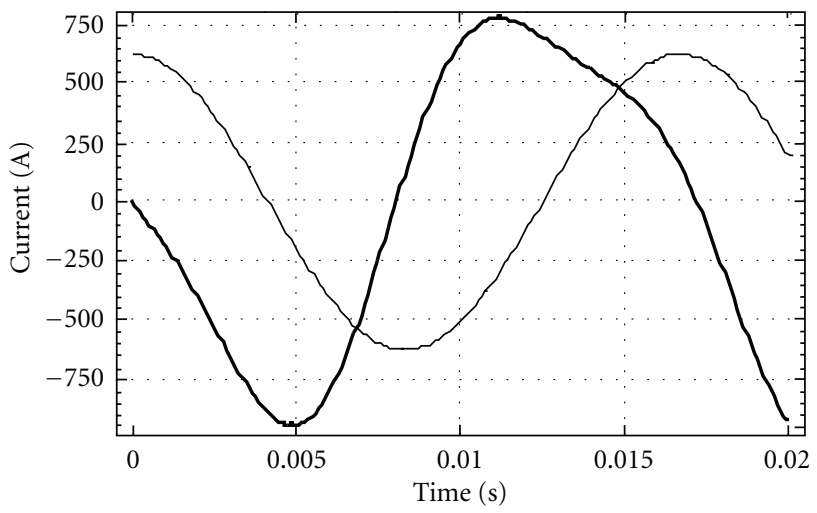

(a)

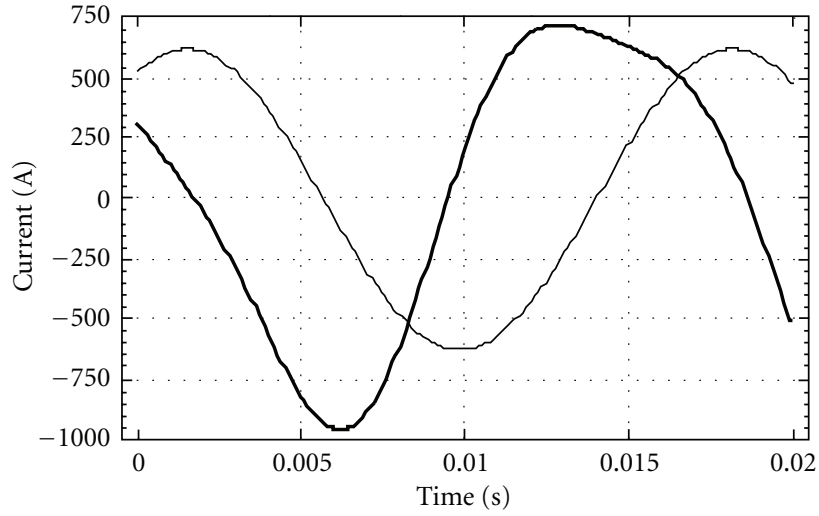

(b)

Figure 7: Waveforms of the total current (thick curves) for the switching of the fourth branch $(k=4)$. The initial voltage is $V_{o}=V_{m}$, $\gamma=0.208$ per unit. The thinner curves represent (to a reduced scale of $1 / 300$ ) the waveform of the source phase voltage. (a) $\alpha=90^{\circ}$ and (b) for the optimal switching angle $\alpha=57.57^{\circ}$.

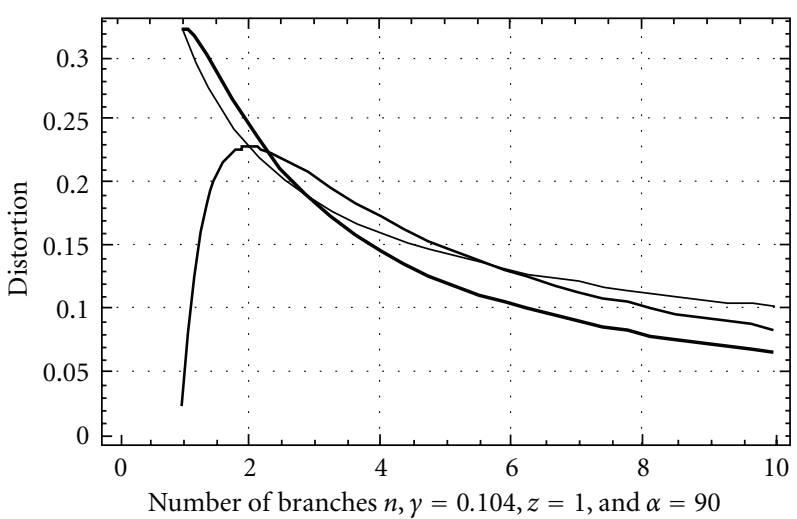

(a)

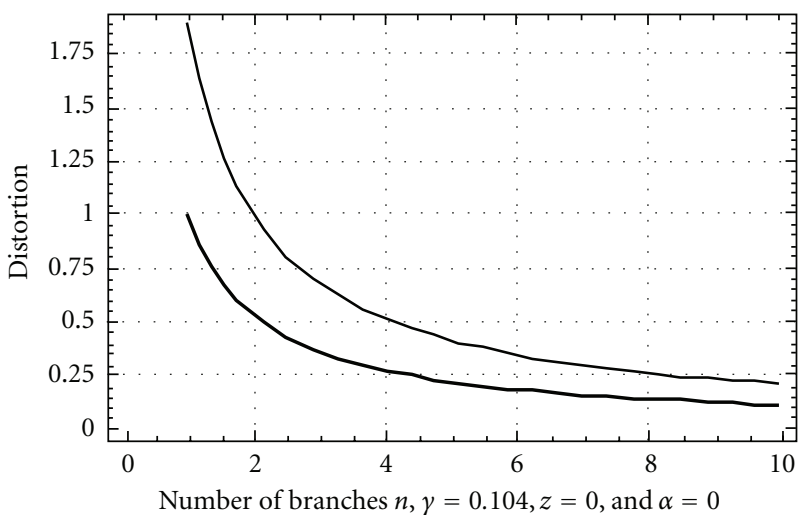

(b)

FIGURE 8: The current distortion factor in per unit versus the total number of branches, $n$ for two cases: (a) initial voltage $V_{o}=V_{m}$, that is, $z=1$ and the switching angle $\alpha=90^{\circ}$. (b) initial voltage $V_{o}=0$, that is, $z=0$ and the switching angle $\alpha=0^{\circ}$. In both cases, the compensator size is $\gamma=0.104$ per unit.

a reduction in the oscillatory current and the corresponding distortion factor in all switching steps except the first. This improvement is $5.78 \%, 8.176 \%$, and $9.589 \%$ for the steps $k=2,3$, and 4 , respectively. These observations are also valid for other per unit compensator ratings $\gamma$. As an example, if $\gamma=0.208$, and considering the last switching step $(k=4)$, the amplitude of the fundamental current will be $839.1 \mathrm{~A}$. If $\alpha=90^{\circ}$ is used, the oscillatory current will be $180.686 \mathrm{~A}$ leading to a distortion factor of 0.215336 . If the optimal angle $\alpha=57.57^{\circ}$ is applied, however, the corresponding values will be $164.083 \mathrm{~A}$ for the oscillatory current and 0.19555 for the distortion factor. Again, there a reduction of $9.19 \%$. This improvement is recognizable in the two plots (a) and (b) in Figure 7 pertinent to the fourth switching step $(k=4)$ for an initial voltage $V_{o}=V_{m}$, that is, $z=1$. They illustrate the waveform of the total current $i(t)$, over 20 millisecond, for the two cases: (a) $\alpha=90^{\circ}$ and (b) for the corresponding optimal switching angle $\alpha=57.57^{\circ}$.

The two plots (a) and (b) of Figure 8 illustrate the effect of the total number of branches $n$ on the oscillatory current component. Each plot includes 3 curves. The thinnest one corresponds to the switching of the first branch (i.e., $k=1$ ). The intermediate one applies for switching the branch of number $k=n / 2$ (this is valid only for even values of $n$ ). The thickest curve is for switching the last branch (i.e., $k=n$ ). In both plots, the per unit compensator size is $\gamma=0.104$. In plot (a) the initial voltage on the switched capacitor is $V_{o}=V_{m}$, that is, $z=1$ and the switching angle $\alpha=90^{\circ}$. In plot (b) the initial voltage is assumed $V_{o}=0$, that is, $z=0$ and the switching angle $\alpha=0^{\circ}$. It should be noted that the thinnest curve in plot (b) is the flat horizontal line of a distortion = 1 per unit, as expected from the analysis of a single-branch TSC. It can be recognized that, with the exception of the case of switching the first capacitor $k=1$ of the case in plot (b), the current distortion decreases steadily with the total number of branches $n$. For example, increasing the number of branches from $n=2$ to $n=6$ will lead to a reduction in the current distortion by a factor of about 2 in case (a) and by a factor of about 3 in case (b). 


\section{Conclusions}

(1) Expressions are derived for the amplitudes of the fundamental current, the oscillatory current and the terminal voltage in a TSC, in terms of the AC network parameters, the TSC total reactive power rating, its total number of branches $n$, the switching step number $k$, and the initial voltage $V_{o}$ of the switched branch as well as on the switching angle $\alpha$.

(2) The procedure is applied to a 3-phase, $60-\mathrm{Hz}, 24-\mathrm{kV}$, 4-branch, and 93.6 MVAr TSC connected to a 230$\mathrm{kV}$ network of a $900 \mathrm{MVA}$ short-circuit level via a 230/24-kV transformer.

(3) The results for the four switching steps assuming initial capacitor voltages equal to the peak supply phase voltage $\left(V_{o}=V_{m}\right)$ and a switching angle $\alpha=$ $90^{\circ}$ indicate that the amplitude of the fundamental current is almost proportional to the switching step number $k$.

(4) Beyond the second switching step, the oscillatory current increases less than proportional with $k$. Its frequency drops from 6.2 times the supply frequency when the first branch is connected $(k=1)$ to half of this value for $k=4$. The largest value of the current distortion is about 0.170 per unit. It occurs when switching the second branch, and the least value is 0.146 and corresponds to $k=4$.

(5) Results of the last switching step $(k=4)$ show the general trend that the current distortion decreases with the compensator rating. For small compensator ratings, the least distortion occurs if the switching takes place at a switching angle close to $\alpha=90^{\circ}$, upon for larger ratings, the minimum distortion can occur over a wider range of $\alpha$.

(6) For small initial capacitor voltages, the least current distortion occurs at the switching angle $\alpha=0$, while the largest distortion results if $\alpha=90^{\circ}$. For initial capacitor voltages close to $V_{m}$, however, the largest distortion occurs at $\alpha=0$. The least distortion will result if $\alpha=67.2^{\circ}$.

(7) The dependence of the optimal switching angle on both the compensator rating and the per unit capacitor initial voltage $z=V_{o} / V_{m}$, for switching each of the four branches, is illustrated. The results for switching the first branch $(k=1)$ are the identical for all compensator ratings, and they follow the relation: $\sin \alpha=V_{o} / V_{m}$. They indicate that the optimal $\alpha$ is $90^{\circ}$ for $V_{o}=V_{m}$. For the switching of the three other branches, the optimal angle will be smaller than $\sin ^{-1}\left(V_{o} / V_{m}\right)$. The deviation increases with both the number $k$ switching step and the capacitor initial voltage $V_{o}$.

(8) Results for the optimal $\alpha$ corresponding to the four switching steps $k$, with $V_{o}=V_{m}$ for three different values of the per unit compensator rating $\gamma$ : $0.030,0.104$, and 0.208 are presented. The optimal $\alpha$ decreases with the TSC rating and with the number $k$ of the switching step.

(9) The values of the fundamental and oscillatory currents as well as the current distortion factor are discussed, if the optimal values of $\alpha$ are used for the four switching steps. Both current components increase approximately proportional to the step number $k$. The distortion factor ranges between 0.163 per unit for $k=2$ and 0.132 for the last step $k=4$. These values are less than the corresponding ones $(0.173$ per unit for $k=2$ and 0.146 for the last step $k=4$ ) resulting from applying $\alpha=90^{\circ}$.

(10) Using the optimal values of $\alpha$ will result in a reduction in the oscillatory current and the corresponding distortion factor in all switching steps except the first. This improvement is $5.78 \%, 8.18 \%$, and $9.59 \%$ for the steps $k=2,3$, and 4, respectively. This advantage increases with the compensator TSC rating.

(11) The effect of the total number of branches $n$ on the oscillatory current is presented for two cases: (a) $V_{o}=$ $V_{m}$ and $\alpha=90^{\circ}$, and (b) $V_{o}=0$ and $\alpha=0^{\circ}$. With the exception of the case of switching the first capacitor $k=1$ of the case (b), the current distortion decreases steadily with the total number of branches $n$. For example, increasing the number of branches from $n=2$ to $n=6$ will lead to a reduction in the current distortion by a factor of about 2 in case (a) and about 3 in case (b).

\section{References}

[1] T. J. E. Miller, Reactive Power Control in Electric Systems, chapter 4, John Wiley \& Sons, New York, NY, USA, 1982.

[2] The IEEE: Tutorial Course, "Applications of staticVAR systems for system dynamic performance," IEEE Publication 87TH0187-5-PWR, 1987.

[3] L. Gyugyi, R. Otto, and T. H. Putman, "Principles and applications of static thyristor-controlled shunt compensators," IEEE Transactions on Power Apparatus and Systems, vol. 97, no. 5, pp. 1935-1945, 1978.

[4] M. M. Saied, "Generalised relations for the current waveforms and the condition for minimum oscillatory currents in multibranch Thyristor-Switched Capacitors," Journal of Electrical and Electronics Engineering, Australia, vol. 12, no. 1, pp. 4248, 1992.

[5] J. Dixon, L. Morán, J. Rodríguez, and R. Domke, "Reactive power compensation technologies: state-of-the-art review," Proceedings of the IEEE, vol. 93, no. 12, pp. 2144-2164, 2005.

[6] G. Olivier, I. Mougharbel, and G. Dobson-Mack, "Minimal transient switching of capacitors," IEEE Transactions on Power Delivery, vol. 8, no. 4, pp. 1988-1994, 1993.

[7] S. Mikhail and M. F. McGranaghan, "Evaluation of switching concerns associated with $345 \mathrm{kV}$ shunt capacitor applications," IEEE Transactions on Power Apparatus and Systems, vol. 1, no. 2, pp. 221-230, 1986.

[8] A. Gelen and T. Yalcinoz, "The behavior of thyristor switched capacitor (TSC) installed in an infinite bus system," in Proceedings of the IEEE International Conference on Computer 
as a Tool (EUROCON '09), pp. 614-617, Petersburg, Russia, May 2009.

[9] J. Peltola, P. Halonen, and A. Kähkönen, "Static var compensator for power oscillation damping," in Proceedings of the IEEE PES Transmission and Distribution Conference and Exposition, New Orleans, La, USA, April 2010.

[10] W. Guo, D. Xu, J. Wu, and L. Wang, "United system of TSC and SVG for reactive power compensation," in Proceedings of the 2nd International Symposium on Power Electronics for Distributed Generation Systems (PEDG '10), pp. 507-511, Hefei, China, June 2010.

[11] P. Chopade, M. Bikdash, I. Kateeb, and A. D. Kelkar, "Reactive power management and voltage control of large transmission system using SVC (static VAR compensator)," in Proceedings of the IEEE Southeastcon, pp. 85-90, Nashville, Tenn, USA, March 2011. 

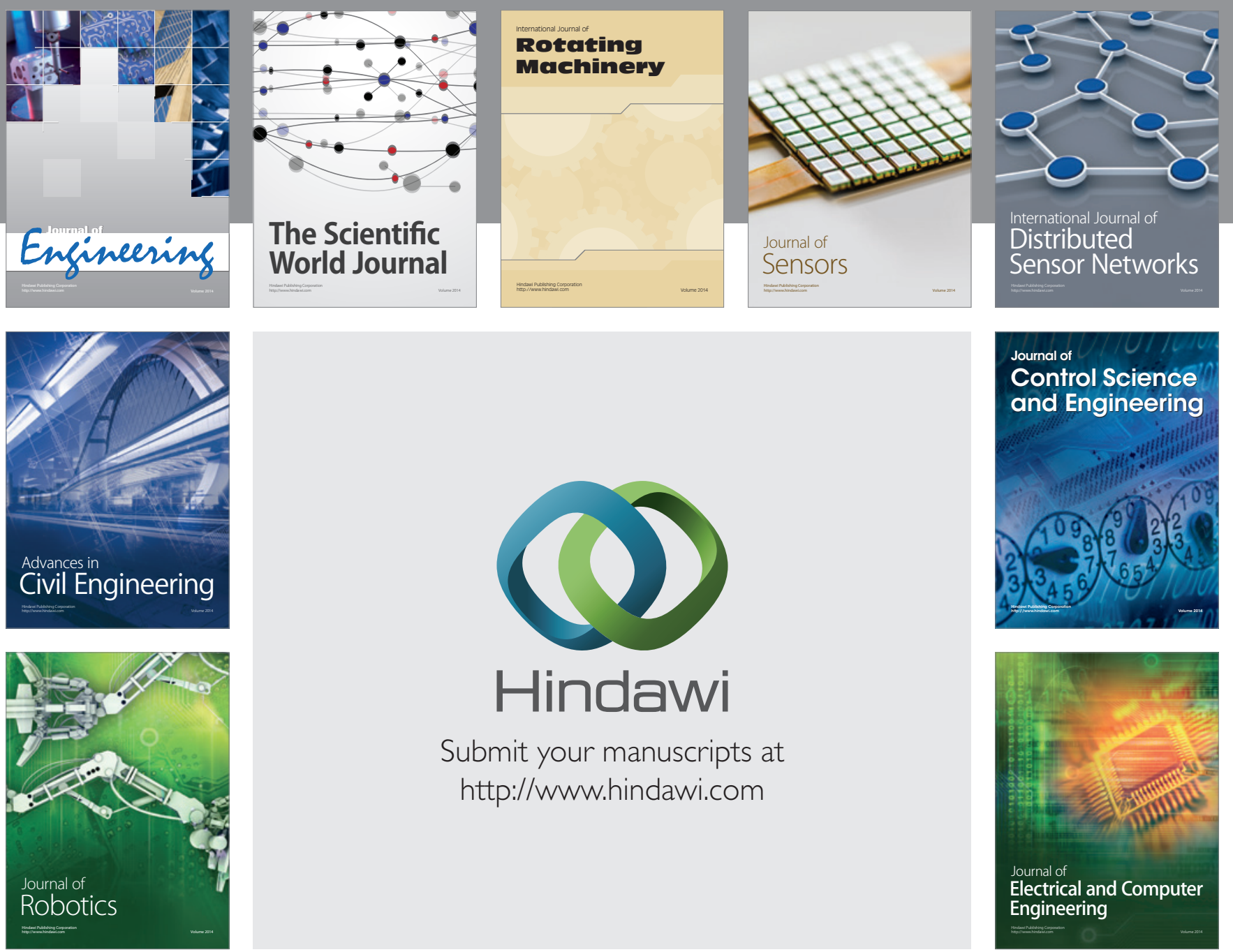

Submit your manuscripts at

http://www.hindawi.com
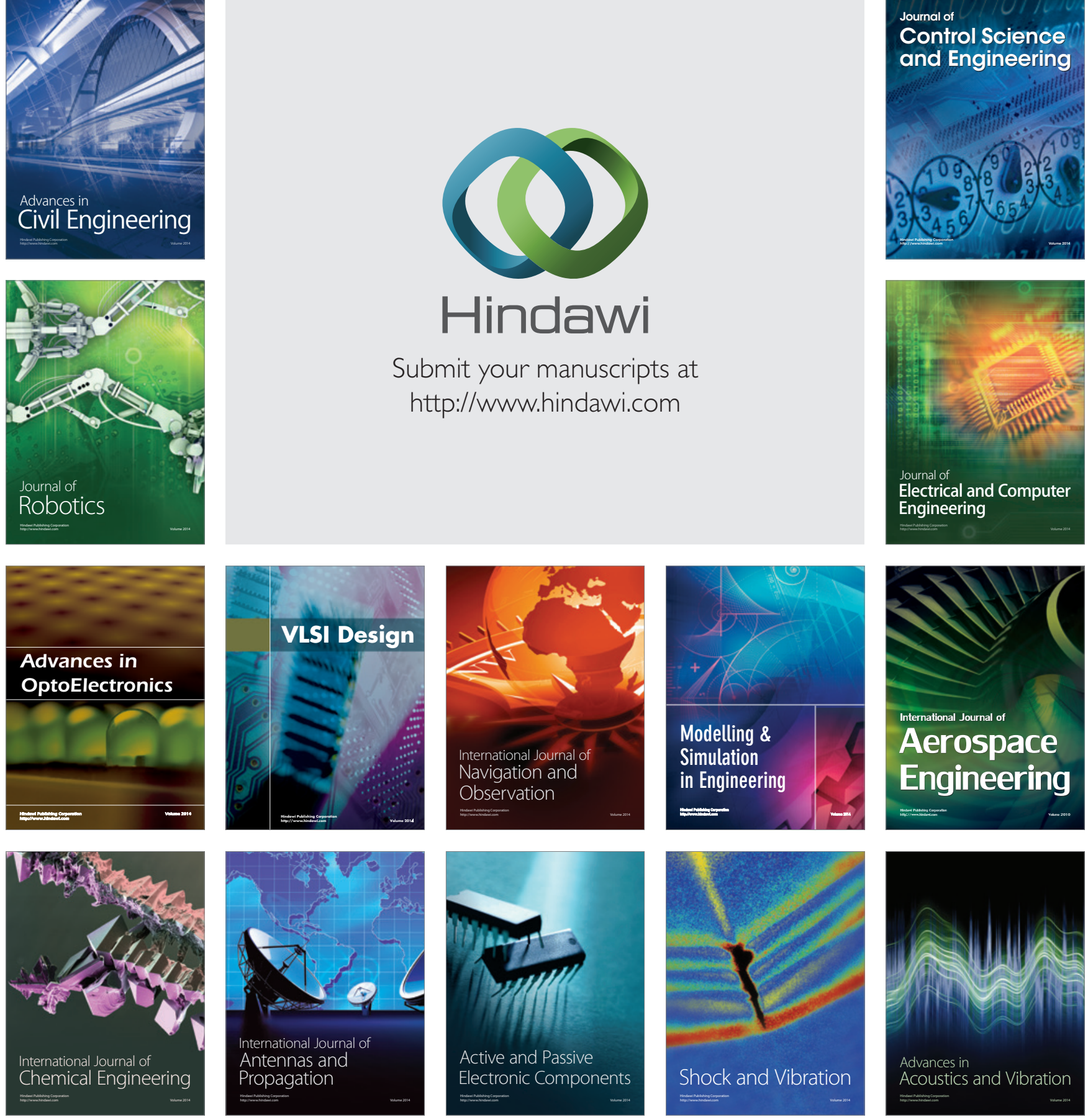